\title{
Active local oscillator power stabilization for a hot electron bolometer heterodyne receiver
}

\author{
D. J. Hayton*a J. R. Gao ${ }^{\mathrm{a}, \mathrm{b}}$, J. W. Kooi ${ }^{\mathrm{c}}, \mathrm{Y}^{\mathrm{a}}$ Ren ${ }^{\mathrm{b}, \mathrm{d}}$, W. Zhang ${ }^{\mathrm{d}}$ and G. de Jange ${ }^{\mathrm{a}}$ \\ ${ }^{a}$ SRON Netherlands Institute for Space Research, Landleven 12, 9747 AD Groningen, The \\ Netherlands; \\ ${ }^{b}$ Kavli Institute of NanoScience, Delft University of Technology, Lorentzweg 1, 2628 CJ Delft, The \\ Netherlands; \\ ${ }^{\mathrm{C} C a l i f o r n i a}$ Institute of Technology, MS 301-17, Pasadena, California, 91125, USA; \\ ${ }^{\mathrm{d}}$ Purple Mountain Observatory (PMO), Chinese Academy of Sciences, 2 West Beijing Road, \\ Nanjing, JiangSu 210008, China
}

\begin{abstract}
We report on the application of a new technique for actively stabilizing the power of a far infrared gas laser as the local oscillator (LO) in a superconducting hot electron bolometer (HEB) heterodyne receiver system at $2.5 \mathrm{THz}$. The technique utilizes PID feedback control of the local oscillator intensity by means of a voice-coil based swing arm actuator placed in the beam path. The HEB itself is used as a direct detector to measure incident LO power whilst simultaneously continuing to function as heterodyne mixer. Results presented here demonstrate a factor of 50 improvement in the measured total power and spectroscopic Allan variance time. Allan times of 30 seconds and 25 seconds respectively are shown for large and small area HEB's with a measured effective noise fluctuation bandwidth of $12 \mathrm{MHz}$. The technique is versatile and can be applied to any LO source and at any LO frequency.
\end{abstract}

Keywords: Hot electron bolometer, Allan variance, terahertz, stability, heterodyne

\section{INTRODUCTION}

In the super terahertz regime (2-6 THz), superconducting hot electron bolometer (HEB) heterodyne mixers offer superior sensitivity when compared to other available mixer technologies such as Schottky diodes and superconductor-insulatorsuperconductor (SIS) receivers [1-2]. However, whilst HEB mixers benefit greatly from a relatively low local oscillator (LO) power requirement, their output stability depends strongly on the fidelity of the LO source which ultimately leads to inferior stability when compared with other devices [3]. This is particularly problematic for applications such as astronomical mapping where sources are weak relative to the background and so require longer integration times. Source-background chopping of the incoming signal is commonly used as a baseline removal technique. However, the switching period can often be as slow as $15-20 \mathrm{sec}$ for a balloon or space instrument if slewing of the entire vehicle is required. Previous studies have demonstrated that improvements in baseline IF stability is achieved through use of a spectroscopic, treatment of receiver output power [4]. This method is very useful and can be applied to practically any heterodyne system however the improvement in stability becomes increasingly limited at greater channel separations due to gain mismatch.

In this work we pay closer attention to the cause of the IF instability and demonstrate a method to reduce it. Previous studies have also demonstrated improved stability by stabilizing the incident LO power in an HEB [5-10]. However until now, they have been either frequency or LO-source specific. Balanced waveguide HEB mixers have demonstrated improved stability but at the expense of complex device design and fabrication [11]. We have developed a method that is simple and can be applied to any LO source and at any frequency with minimal modification to the receiver. Some of the results presented here were first published in Appl. Phys. Lett. 2012 by Hayton et al, [12].

*d.j.hayton@sron.nl; tel: +31 503639363

Millimeter, Submillimeter, and Far-Infrared Detectors and Instrumentation for Astronomy VI, edited by Wayne S. Holland, Jonas Zmuidzinas, Proc. of SPIE Vol. 8452

$84520 Z$ @ 2012 SPIE · CCC code: 0277-786X/12/\$18 - doi: 10.1117/12.925851 


\section{HEB DEVICE AND STABILIZATION METHOD}

\subsection{HEB device}

Phonon cooled NbN HEB mixers of two different sizes were studied for this work enabling comparisons of relative stability between large and small volume devices. The larger device has dimensions $0.4 \times 4 \mu \mathrm{m}^{2}$ and the smaller device is $0.2 \times 2 \mu \mathrm{m}^{2}$. Optimum LO pumping power is $450 \mathrm{nW}$ and $120 \mathrm{nW}$ respectively for the large and small area HEB's. The HEB chip mounts directly to a $10 \mathrm{~mm}$ diameter, uncoated high resistivity Si lens which, in turn, is mounted in a mixer block on the $4.2 \mathrm{~K}$ stage of a liquid He cryostat. The local oscillator is provided by a far infrared (FIR) gas ring laser that is pumped by a commercial DEOS $\mathrm{CO}_{2}$ laser and tuned to the $2.523 \mathrm{THz}$ methanol line. The beamsplitter is a $3 \mu \mathrm{m}$ thick low loss mylar film. Both devices were measured using a vacuum hot/cold load setup as shown in figure 1 . In this configuration, the beamsplitter and hot/cold sources share a common vacuum space with the mixer. This setup reduces signal optical loss considerably since there is no need for a cryostat window and no atmospheric absorption between the source and the mixer.

The HEB consists of a superconducting thin film that bridges two normal metal contacts. These contact leads also serve as an antenna for quasi-optical coupling of radiation into the device. A combination of both electrical bias and LO optical pumping maintain a temperature distribution of hot electrons across the superconducting film which creates a region of normal conductor in the centre of the bridge [13]. Modulation of this hot spot length at the IF occurs as a result of heterodyne mixing. Any change in total incident power will therefore directly influence mixer performance parameters such as sensitivity, conversion gain, IF bandwidth etc. The total power dissipated in the bridge, $\mathrm{P}_{\text {total }}$, is given by:

$$
\mathrm{P}_{\text {total }}=\mathrm{P}_{\mathrm{LO}}+\mathrm{P}_{\text {bias }}+\mathrm{P}_{\text {signal }}
$$

Where $\mathrm{P}_{\mathrm{LO}}$ is the absorbed LO power, $\mathrm{P}_{\text {bias }}$ is the electrical bias power dissipated across the bridge and $\mathrm{P}_{\text {signal }}$ is the absorbed optical signal. Since the bias is constant and the signal is generally small, the LO is the dominant source for potential instability. Without correction, any drift in LO power can accumulate with time so that the device is no longer operated at a constant operating point. This can also create additional problems over longer timescales in terms of instrument calibration.

It should also be noted that large changes in the signal power that can result from broadband hot / cold source chopping, such as that used for Y-factor measurements in the lab, can also be an issue for small area HEB mixers. In this case, source direct detection can occur whereby the change in signal can be large enough to influence the HEB bias condition leading to large non-linearity in the output.

\subsection{Stabilization method}

LO amplitude drift and source direct detection can both be reduced by manually adjusting the LO intensity to maintain a constant total power in the HEB ( $\mathrm{P}_{\text {total }}$ in eq. 1). Previous reports have shown that is possible to add electrical feedback to particular LO sources in order to automate this adjustment [5-10]. Another method demonstrates addition of a third optical source at a frequency outside of the device bandgap in order to supplement the LO [14]. These methods have demonstrated improved stability with varying success however they are all tailored to a particular LO source/HEB/frequency combination. 


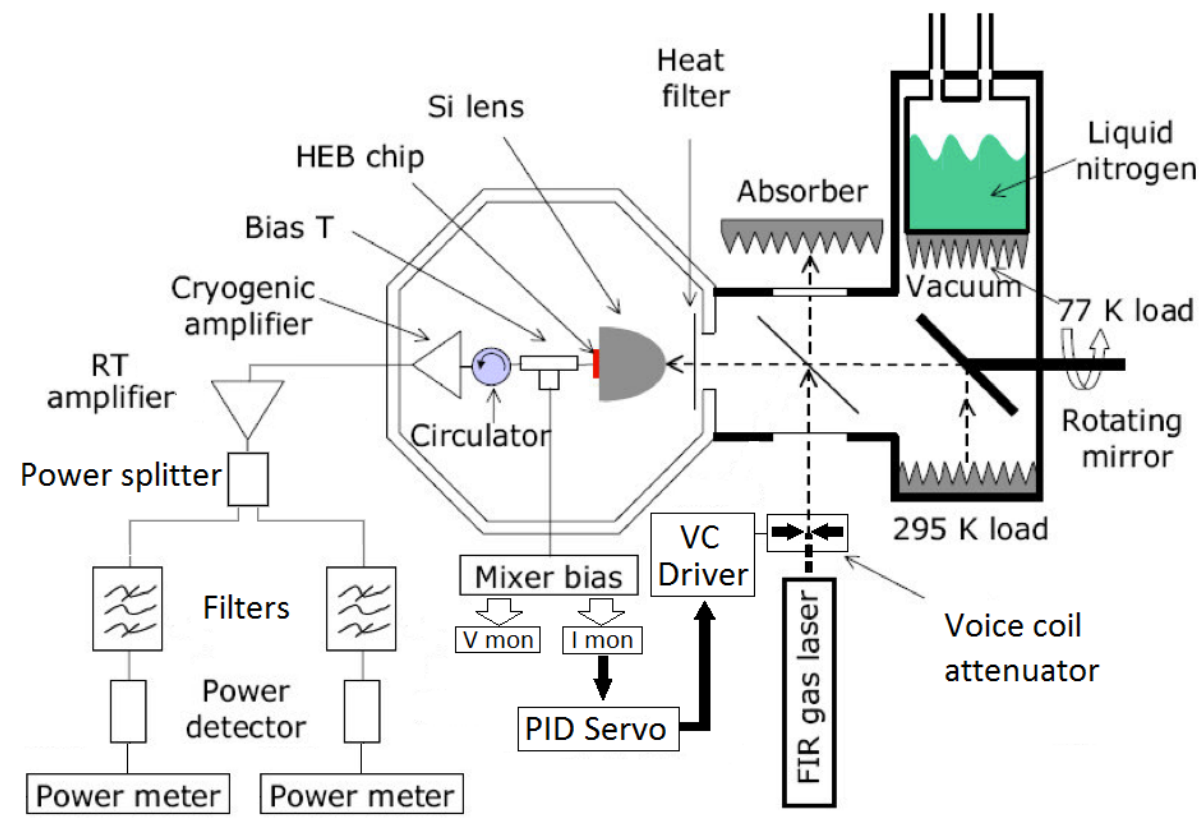

Figure 1 Schematic showing the system setup for voice coil stabilized Allan variance measurements in spectroscopic configuration.

Initial attempts at stabilizing the LO used a similar feedback loop with a computer controlled polarizer grid to adjust or maintain the incident laser intensity. Although this approach also works, it was found that angular resolution and rotation speed of the polarizer drive mechanism were not sufficient to correct for LO noise at higher AM frequencies. In a new approach to this problem, we have developed a variable optical attenuator based on a voice-coil swing arm actuator [15], which is placed in the LO beam path to provide rapid control of the transmitted $\mathrm{THz}$ beam intensity. This actuator quickly and accurately positions an absorber such that it interrupts part of the $\mathrm{THz}$ beam. The degree to which this absorber is inserted is a function of the applied current and so allowing for rapid changes in beam intensity at speeds beyond $1 \mathrm{kHz}$. Another advantage to this method is the full range of attenuation available - from 0 to $100 \%$ effective transmission. This attenuator, in conjunction with the direct detection output of the HEB and a proportional - integral differential (PID) controller with $10 \mathrm{kHz}$ bandwidth, completes the feedback loop.

Since this approach uses a dedicated optic element for LO intensity control, the stabilization scheme is independent of LO source-type and frequency and so can be applied to a large range systems without modification to the mixer device or IF chain. We have demonstrated its use with several LO sources including: FIR gas laser, backward wave oscillator (BWO), solid state source and QCL over a frequency range of 1.6 to $5.3 \mathrm{THz}$.

Since only the incident total power of the LO is important, changes to the beam shape caused by physical interruption of the beam have no negative effects on device mixer performance. To verify this, receiver noise temperature has been measured for a single HEB using both a rotating polarizer and voice coil actuator with identical results. This can be seen in Figure 2 in the form of a Y-factor measurement using a standard hot/cold load method [16] and fixed bias settings. In the non-stabilized time series, drift and higher frequency noise is apparent. When stabilization is applied through use of a motor driven polarizer grid, the drift is removed. In the case of the voice coil stabilized system, both drift and higher frequency noise is removed whilst the $1.1 \mathrm{~dB}$ Y-factor remains unchanged. 

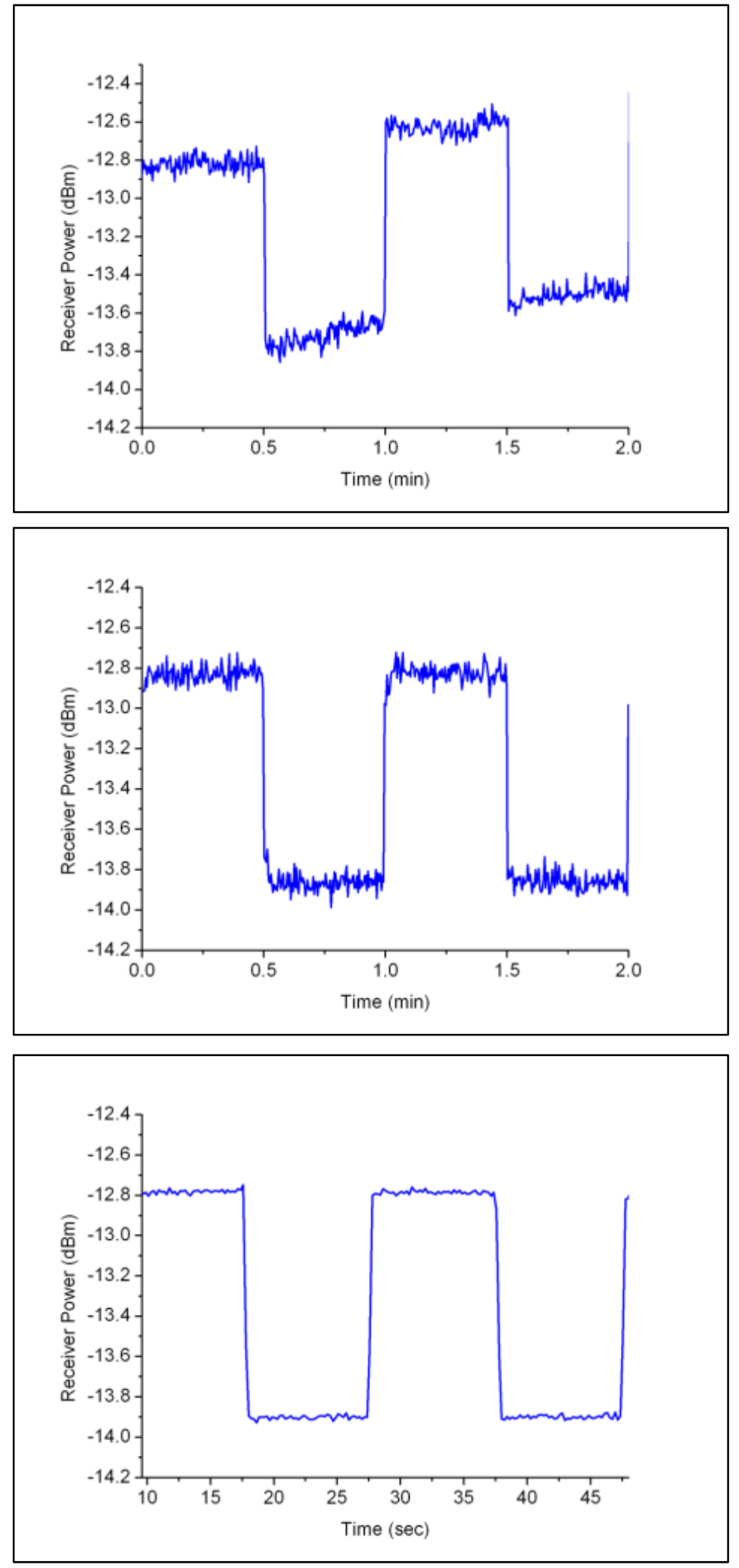

Figure 2 Hot/cold receiver output power time-series data for large area HEB at optimum bias. (top) No stabilization. (center) Stabilized with the motorized polarizer. (bottom) Stabilized with the voice coil. 


\section{RESULTS}

\subsection{Allan variance}

To quantify the effectiveness of the stabilization method we measure Allan variance [17] in two configurations; total power (continuum) and spectroscopic (spectral differential). Allan variance is a well-known tool for measuring and characterizing the stability of a system. It is known that random, white noise, integrates down with time according to the radiometer equation [18]. The finite stability of a real system eventually deviates from the radiometer equation at an integration time known as the Allan variance time. The quoted system Allan time thus refers to the optimum useful integration time for a given noise fluctuation bandwidth. In the case of a drift noise limited source, the Allan time is defined as the integration time in which the variance reaches a clear minimum. However, on occasions when a clear minimum is not present, as is the case with a source displaying high inverse-frequency noise, the Allan time defined as the time at which the variance deviates by more than $20 \%$ from the radiometer equation.

In the literature, HEB Allan times are quoted at different bandwidths and often referred to only by IF input signal bandwidth. However, due to time inefficiency in power meter sampling and power meter internal noise contribution, the measured effective noise fluctuation bandwidth differs from the IF input signal bandwidth, and therefore needs to be characterized. In the case of the measurements presented here, the IF input signal bandwidth is defined by an $80 \mathrm{MHz}$ tunable filter whereas the measured effective noise fluctuation bandwidth is $12 \mathrm{MHz}$.

A schematic of the setup describing the Allan variance measurement is shown in figure 1. The IF signal is first amplified by a Berkshire 1-2 GHz cryogenic amplifier and then further amplified by two MITEQ room temperature amplifiers before being filtered by an $80 \mathrm{MHz}$ bandwidth $(-3 \mathrm{~dB})$ tunable $1-2 \mathrm{GHz}$ bandpass filter (verified independently). To derive the total power instrumental stability, the IF signal is sampled by a fast Agilent E4418B power meter. In the spectroscopic (dual channel) configuration, the IF signal is split after the first room temperature amplifier using a power splitter. The parallel IF signals are then filtered $(80 \mathrm{MHz})$ and fed to two identical (Agilent E4418B) power meters. A channel separation of $500 \mathrm{MHz}$ is used in spectroscopic mode with filters set to $1.25 \mathrm{GHz}$ and $1.75 \mathrm{GHz}$. IF baseline removal is achieved using the method identified in Tolls et al [19]. Power meters are read out sequentially in a fast 200 samples/sec mode, in which all power meter automated features and signal averaging/filtering are disabled. It should be noted that the reduced power present in the dual channel setup results in a smaller effective noise fluctuation bandwidth due to the noise floor limitations in the power meter described above. In all instances, at least 40 minutes of contiguous data is acquired for each Allan variance plot and the entire data set is used each time. Measurements were taken in a temperature controlled lab during the evening for optimum conditions. The IF amplifiers were left to settle for several hours before beginning measurements. As was the FIR laser.

In order to independently assess the stability of the IF chain (IF amplifier/filter/power meter) without noise contribution from the HEB, we first place the HEB in a fully superconducting state by applying neither electrical bias nor LO power. In this state, the IF amplifier chain is effectively in an input short mode. The recorded Allan variance in this state is very long at around 100 seconds spectroscopic. From this we conclude that the IF amplifier chain is not a significant source of system instability.

Figure 3 shows the effectiveness of the stabilization technique by comparing non-stabilized and stabilized Allan variance for the large device. Resulting Allan variance data is shown for stabilized, non-stabilized, total power and spectroscopic measurement of a large device as well as stabilized spectroscopic data for a small volume device. The data shows stability for each device operating at its optimum bias point and LO power. It can be seen that in the case of nonstabilized data, the spectroscopic method improves the Allan time by a factor of 10 which is similar to that reported in the literature. However, we can also show that, by applying the stabilization method, a further improvement of a factor of 50 is gained. This leads to a total Allan time at optimum bias in excess of $30 \mathrm{sec}$ at the $12 \mathrm{MHz}$ effective bandwidth. Allowing for bandwidth, this is considerably longer than other reported HEB Allan times above $1.5 \mathrm{THz}$. Indeed, in the case of a standard backend spectrometer with $1.6 \mathrm{MHz}$ noise spectral bandwidth this Allan time would equate to around $75 \mathrm{sec}$. 


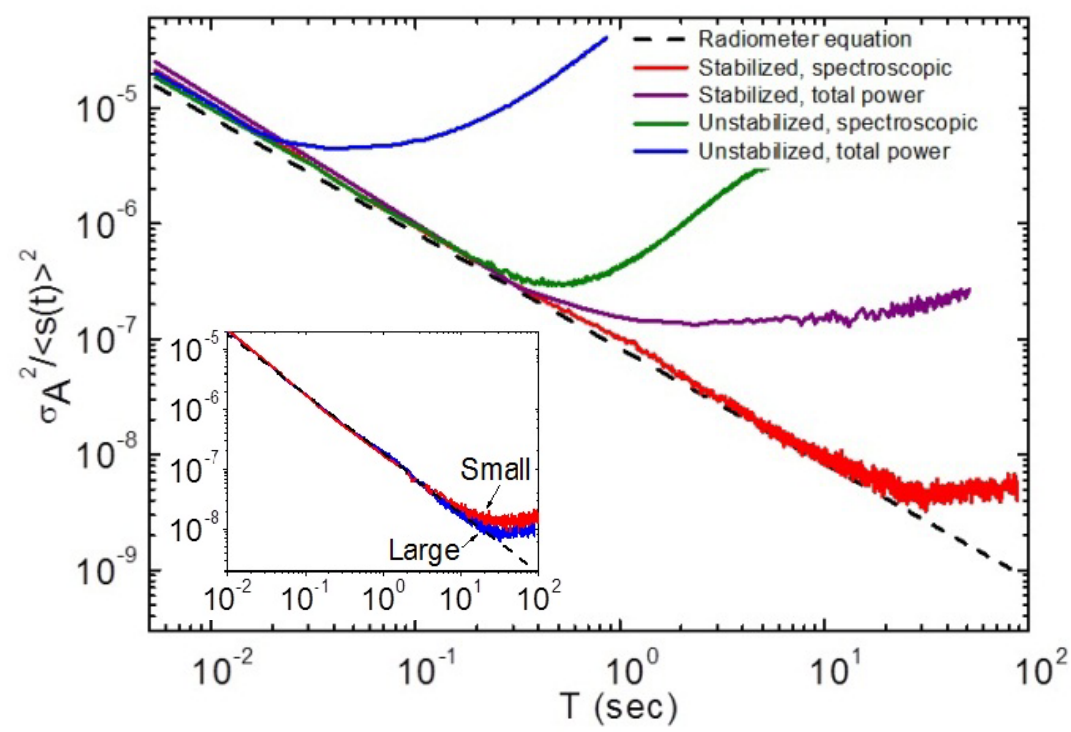

Figure 3 Measured (normalized) Allan variance curves using dual channel setup for large area HEB stabilized, unstabilized, total power and spectroscopic configurations at optimum bias. In the inset; comparison of spectroscopic Allan variance for large area $0.4 \mathrm{x}$ $4 \mu \mathrm{m} 2$ and small area $0.2 \times 2 \mu \mathrm{m} 2 \mathrm{HEBs}$. The radiometer equation for an effective noise fluctuation bandwidth of $12 \mathrm{MHz}$ is shown in both plots [12]

Figure 4 shows the influence of bias voltage on HEB stability for both the stabilized and unstabilized case. For the unstabilized case, stability is directly influenced by absorbed electrical power. As the voltage increases, the HEB becomes saturated. At very high bias, the HEB becomes almost fully resistive and thus the conversion gain becomes small with little sensitivity to LO power fluctuation, resulting in a long Allan time as expected. In contrast, with stabilization applied, the Allan minimum is approximately constant with bias voltage up to a few $\mathrm{mV}$, suggesting that the LO is no longer the dominant source of instability. The stabilization scheme is not shown for the high bias case since the HEB direct detector sensitivity is also significantly reduced. Reduced direct detection sensitivity also occurs at low HEB bias. This is evident in Figure 4 where excess noise is present at the lower bias setting.

We attribute at least a part of the remaining instability to a noise limitation in our bias supply voltage and current monitor readout circuit. In this case, any error in the measurement of the HEB mixer current will cause the feedback loop to incorrectly adjust the LO power. i.e. bias readout noise is transferred into LO intensity noise. The nature of this noise is likely to be $1 / \mathrm{f}$ since it is caused by the relatively poor noise performance of output ground isolation amplifiers within the bias supply circuit. This is also in agreement with the increased $1 / \mathrm{f}$ nature of the stabilized system relative to the unstabilized system shown Figure 3 although it is difficult to differentiate between bias supply noise and intrinsic HEB noise. The magnitude of the noise limitation has been estimated from power spectral density (PSD) measurements of the HEB voltage and current bias monitor outputs as shown in figure 5. The data clearly show that the feedback loop removes a very large noise component and is effective up to about $200 \mathrm{~Hz}$. It also shows $1 / \mathrm{f}$ noise present in the voltage monitor output. Using this data to characterize the readout noise, we estimate that the level of bias supply induced LO noise is around $25 \mathrm{~dB}$ below the level of non-stabilized LO noise $(<10 \mathrm{~Hz})$. An upgrade of our bias supply is expected to reduce current measurement noise and further improve the effectiveness of this stabilization method. If sufficient removal of instrumental instability could be achieved then it may be possible to use this technique to get a better understanding of the intrinsic stability of the HEB. 


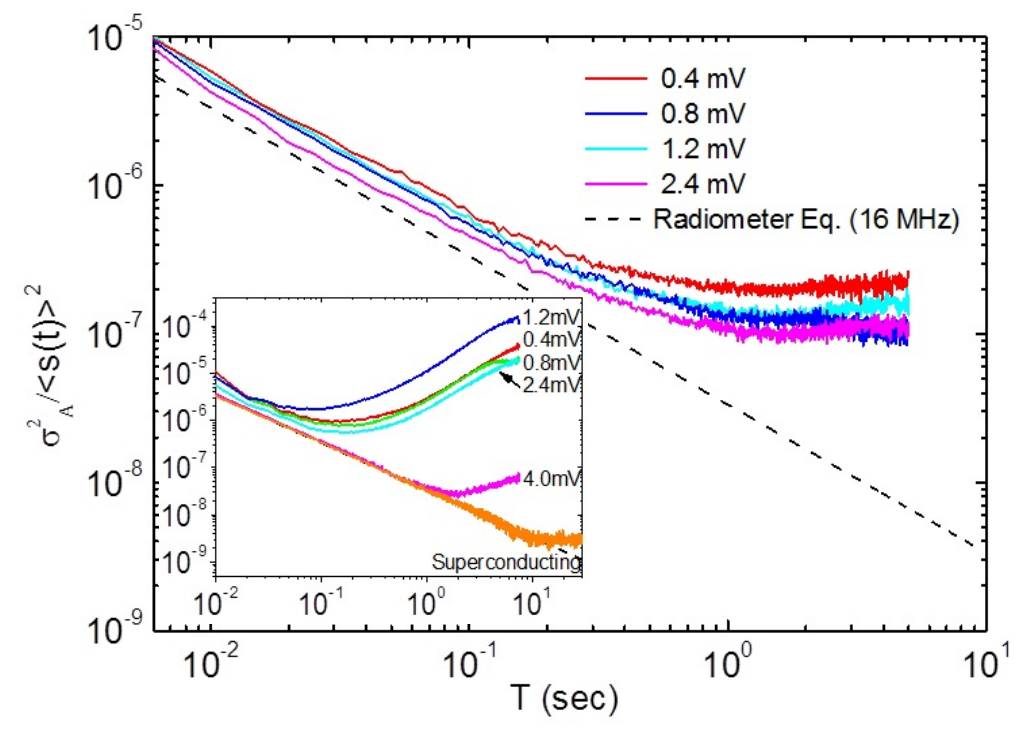

Figure 4 Measured (normalized) total power Allan variance using single channel IF setup. Stabilization is applied for several HEB bias voltages. The $0.8 \mathrm{mV}$ curve represents optimum bias for this device. In the inset, measured total power Allan variance curves without stabilization applied. The radiometer equation for a noise fluctuation bandwidth of 25 and $30 \mathrm{MHz}$ is shown in the main and inset plots respectively. All data sets are measured with a large area HEB [12].

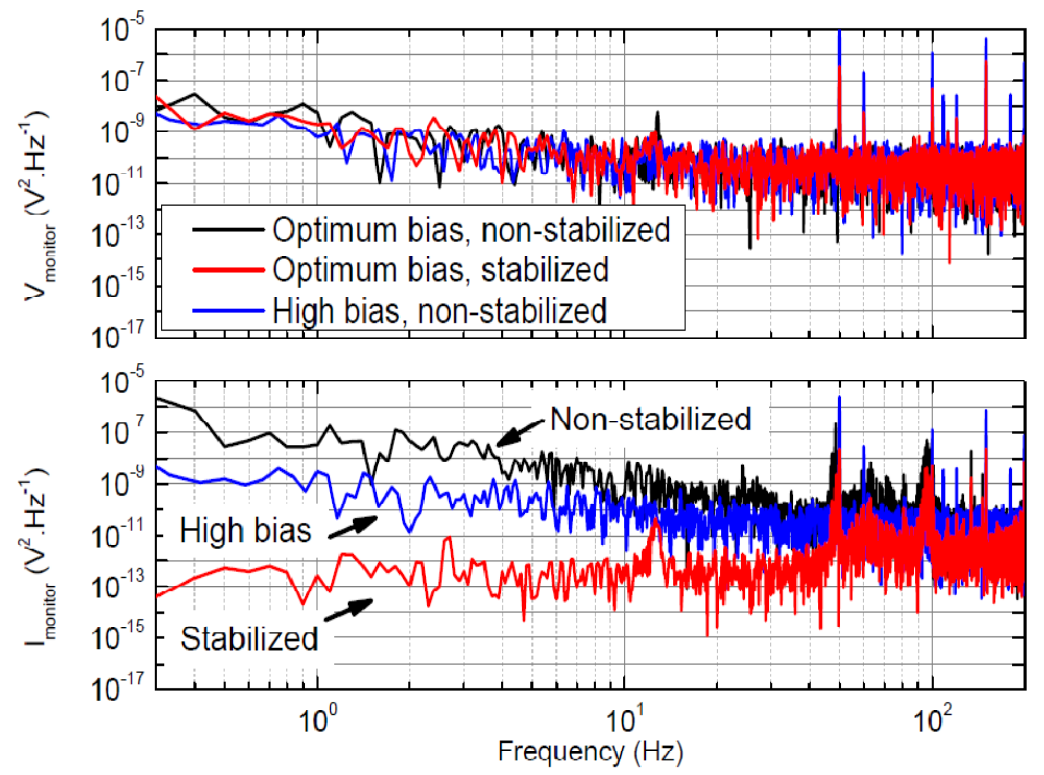

Figure 5 Power spectral density plots for HEB bias voltage monitor (upper panel) and current monitor (lower panel) outputs, with and without stabilization applied for a large area HEB. The HEB is constant voltage biased at optimum $(0.8 \mathrm{mV})$ and with optimum LO power applied. In addition, data is shown for an HEB with high bias voltage applied $(8 \mathrm{mV})$ to demonstrate the $1 / \mathrm{f}$ noise limitation of our bias supply readout circuit [12].

\subsection{Lab applications}

This stabilization technique and specifically, the ability to precisely control both voltage and current HEB bias points has already demonstrated its usefulness in the lab by simplifying standard HEB characterization techniques for receiver noise temperature and IF bandwidth determination. In the first case, the method for determination of receiver noise temperature using a fixed LO power and swept $\mathrm{V}_{\text {bias }}$ has strong susceptibility to LO power drift since any change in LO power that occurs between hot and cold sweeps will cause error in the resulting noise temperature. In addition to this, 
signal direct detection is often an issue when using this method, particularly for small area HEB's. We have shown that it is possible to make these measurements using a stabilized LO by first measuring an I-V curve at a chosen LO power, fitting a poly nominal to it and then using that curve to determine the current set point at each bias voltage throughout the sweep. The advantage here is that the HEB current and voltage bias conditions are exactly replicated for each of the hot and cold sweeps regardless of LO noise. Secondly, stabilization of the HEB bias point is very advantageous when measuring gain and noise IF bandwidth in the lab since the mixer conversion gain is maintained constant regardless of the measurement duration.

\section{CONCLUSION}

We have demonstrated improved HEB IF stability using a novel voice coil based feedback method to stabilize the HEB bias current. This method shows improvements in Allan time of up to a factor 50 in both total power and spectroscopic configurations. A long HEB Allan time of $30 \mathrm{sec}$ has been measured at an effective noise fluctuation bandwidth of 12 $\mathrm{MHz}$ using the particularly unstable pairing of a gas laser with an HEB. The technique is independently of LO frequency or LO source type and can be added to an existing HEB receiver with minimal modification. We have successfully applied this technique to stablize the amplitude of a THz quantum cascade laser as a LO [20]. The enhanced stability afforded by the proposed technique will make astronomical observing routines such as 'on the fly mapping (OTF)' significantly more efficient as fewer off source reference scans will be needed.

\section{ACKNOWLEDGMENTS}

We acknowledge T.M. Klapwijk for his helpful discussions, M. Hajenius for fabricating the HEBs, L. de Jong for his technical support and G. Gol'tsman's group at MSPU for provision of NbN films. The work was supported by NWO, KNAW China Exchange Programme, the AMSTAR+ of RadioNet under FP7, and NATO SfP

\section{REFERENCES}

[1] J. Zmuidzinas and P.L. Richards, Proc. IEEE 92, 1597 (2004).

[2] W. Zhang, P. Khosropanah, J.R. Gao, E.L. Kollberg, K.S. Yngvesson, T. Bansal, R. Barends, and T. M. Klapwijk, Appl. Phys. Lett. 96, 111113 (2010).

[3] J.W. Kooi, G. Chattopadhyay, M. Thielman, T.G. Phillips, and R. Schieder, Int J. IR and MM Waves, 21,5 (2000)

[4] V. Ossenkopf, Astronomy and Astrophysics, 479, 915-926 (2008)

[5] J.W. Kooi, J.J.A. Baselmans, A. Baryshev, R. Schieder, M. Hajenius, J.R. Gao, T.M. Klapwijk, B. Voronov, and G. Gol’tsman, J. App. Phys. 100, 064904 (2006)

[6] S. Cherednichenko, V. Drakinskiy, T. Berg, P. Khosropanah, and E. Kollberg, Rev. Sci. Instrum. 79, 034501 (2008)

[7] R. Schieder, C. Kramer, Astronomy and Astrophysics, 373, 746-756 (2001)

[8] M.C. Wiedner, G. Wieching, F. Bielau, K. Rettenbacher, N.H. Volgenau, M. Emprechtinger, U.U. Graf, C.E. Honingh, K. Jacobs, B. Vowinkel, K.M. Menten, L. Nyman, R. Gusten, S. Philipp, D. Rabanus, J. Stutzki, F. Wyrowski, Astronomy and Astrophysics, 434, L33-L36 (2006)

[9] J. Chen, Y. Jiang, M. Liang, L. Kang, B.B. Jin, W.W. Xu, P.H. Wu, IEEE Trans. App. Supercond. 21, 3 (2011)

[10] R. Zannoni, K.S. Yngvesson, Proc. IRMMW-THz, 1-2 (2008)

[11] D. Meledin, A. Pavolotsky, V. Desmaris, I. Lapkin, C. Risacher, V. Perez, D. Henke, O. Nystrom, E. Sundin, D. Dochev, M. Pantaleev, M. Fredrixon, M. Strandberg, B. Voronov, G. Gol'tsman, V. Belitsky, IEEE Trans. Mic. Theory Tech. 57, 1 (2009)

[12] D. J. Hayton, J. R. Gao, J. W. Kooi, Y. Ren, W. Zhang and G. de Lange, Appl. Phys. Lett. 100, 081102 (2012)

[13] R. Barends, M. Hajenius, J.R. Gao, and T.M. Klapwijk, Appl. Phys. Lett. 87, 263506 (2005)

[14] S. Ryabchun, C.-Y.E. Tong, R. Blundell and G. Gol'tsman, IEEE Trans. App. Supercond. 19, 1 (2009)

[15] G.P. Gogue, J.J. Stupak, PCIM Conference (1989)

[16] P. Khosropanah, J. R. Gao, W. M. Laauwen, M. Hajenius, and T. M. Klapwijk, Appl. Phys. Lett. 91, 221111 (2007).

[17] D.W. Allan, Proc. IEEE, 54, 2 (1966)

[18] J.D. Kraus, Radio Astronomy (McGraw-Hill, New York, 1966) 
[19] V. Tolls, R. Schieder, G. Winnewisser, Experimental Astronomy, 1, 101 (1989)

[20] Y. Ren, D.J. Hayton, J.N. Hovenier, M. Cui, J.R. Gao, T.M. Klapwijk, S.C. Shi, T-Y. Kao, Q. Hu, and J. L. Reno, This SPIE proceedings 